Symposium: 2015 Meetings of the Musculoskeletal Tumor Society and the International Society of Limb Salvage

\title{
Editorial Comment: Symposium: 2015 Meetings of the Musculoskeletal Tumor Society and the International Society of Limb Salvage
}

\author{
John H. Healey MD, FACS
}

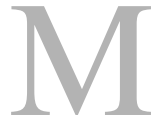

usculoskeletal oncology encompasses a widening array of investigation, and an ever-expanding spectrum of disciplines are involved in our research. Molecular genetics, developmental biology, epidemiology, prognostication, environmental pathogens, imaging advances, surgical strategies, pathological (re)classification, reconstruction methods, implant fixation,

The author certifies that he, or any members of his immediate family, has no funding or commercial associations (eg, consultancies, stock ownership, equity interest, patent/ licensing arrangements, etc.) that might pose a conflict of interest in connection with the submitted article.

All ICMJE Conflict of Interest Forms for authors and Clinical Orthopaedics and Related Research ${ }^{\mathbb{R}}$ editors and board members are on file with the publication and can be viewed on request.

The opinions expressed are those of the writers, and do not reflect the opinion or policy of $C O R R^{\mathbb{R}}$ or The Association of Bone and Joint Surgeons ${ }^{\mathbb{R}}$.

J. H. Healey MD, FACS ( $\square)$

Orthopaedic Service, Department of Surgery, Memorial Sloan Kettering

Cancer Center, 1275 York Avenue, New

York, NY 10065, USA

e-mail: healeyj@mskcc.org new devices, adjuvant therapies, drug development, operative complications, functional outcome, and quality of life characterize the presentations and discussions at the meetings of the Musculoskeletal Tumor Society (MSTS) and the International Society of Limb Salvage (ISOLS). The manuscripts in this symposium make useful contributions to our understanding of the causes, behaviors, and consequences of musculoskeletal tumors.

However, we face a number of ongoing problems as a specialty that call for our attention. Owing to the rarity of the cancers that we treat, it is difficult to accrue large patient numbers. Most reports are small retrospective series. Studies often are underpowered. High-level evidence remains scant. Articles commonly conclude by advocating for more studies and multi-institutional randomized trials. How can we accomplish this, particularly given the crises of research prioritization, funding, and surgeon commitment? While it is easy to blame political agencies and regulators for our limitations, they are only part of the issue. For answers, we need to look to ourselves. I will

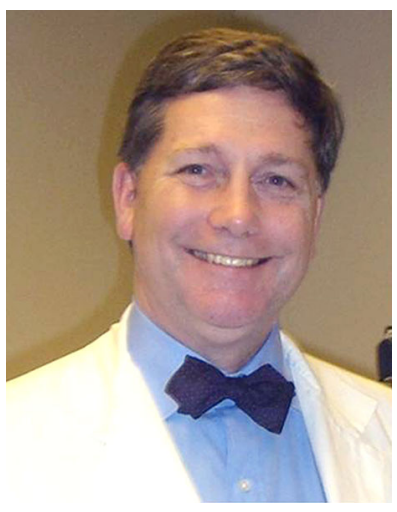

John H. Healey MD, FACS

highlight a few of the obstacles and solutions that have sprung from the work of the MSTS and ISOLS.

Research priorities need to be set and defended as we work with other specialties and focus our own efforts. Modern biomedical research is a team sport. Multidisciplinary care has become the norm for most cancers and even some benign tumors. Examples include denosumab for giant cell tumor of bone and Pexidartinib (Daiichi-Sankyo, Parsippany, NJ, USA) for giant cell tumor of tendon sheath. Studies of these disease-modifying agents are driven by drug companies and medical oncologists. The role of 
Symposium: 2015 Meetings of the Musculoskeletal Tumor Society and the International Society of Limb Salvage

the orthopaedic surgeon is often seen as tangential. In actuality, the orthopaedic surgeon's role is essential. First, orthopaedic involvement at all levels is needed to help design a clinically relevant study. Who knows more about the disease than we do? Second, orthopaedic participation is needed to accrue patients since most of the patients still present to the orthopaedic surgeon for musculoskeletal care. Even when the effectiveness of a new drug is the principal experimental outcome, orthopaedic questions should still be included as secondary or exploratory outcome measures. By doing so, we can set the stage for future sophisticated orthopaedic research in the oncology population. The orthopaedic oncology agenda was the recent focus of a Delphi panel effort led by Michele Ghert MD, FRCSC in conjunction with the 2016 MSTS annual meeting. The forthcoming work product of this effort will be important to guide priorities in our field.

Funding is an ever-present problem. National public funding is slim. A majority is allocated to fund the relevant cooperative group, the Children's Oncology Group, within which R. Lor Randall is the orthopaedic leader. Our orthopaedic "home," the National Institute of Arthritis and Musculoskeletal and Skin Diseases (NIAMS), had a payline in the 13th percentile for new NIH Research Grant Program applications in fiscal year 2016. A minority of the funded grants are in the orthopaedic domain, and NIAMS rarely accepts applications in the oncology field. The National Cancer Institute (NCI) does fund work in musculoskeletal oncology, but most of what they fund is in the sphere of metastatic disease. Few NCI grants have ever been awarded in the orthopaedic surgery domain. Stepping into this void, the Orthopaedic Research and Education Foundation (OREF) has been a prime funder of orthopaedic oncology research, supporting six new oncology grants in 2016 alone (one USD 98,000, three USD 20,000, and two USD 5000 grants), in addition to funding interesting related work in infection, tendon healing, and other relevant fields. The OREF's major clinical research grant has been awarded to two randomized clinical trials in oncology in recent years: The PARITY trial examining different schedules of prophylactic antibiotics in knee/femur reconstruction, and a randomized study of intramedullary nail fixation versus long-stemmed hemiarthroplasty for subtrochanteric pathological fractures. Other notable developments include the MSTS and OREF working with the Musculoskeletal Transplant Foundation to fund clinical and basic research relevant to musculoskeletal transplantation. Several other initiatives have helped focus our research efforts. The MSTS has chosen to aggregate funds and sponsor multi-institutional projects of clinical relevance in an effort to make larger awards for high-quality proposals, rather than supporting local investigator-initiated projects. That task has fallen to local and national grassroots philanthropic efforts, such as Jake's Reindeer Race organized by the Maynard Childhood Cancer Foundation, Matthew DiCaprio's Team Sarcoma 5K Run/Walk in Albany, New York, and many other institutionally-based development efforts, which have provided indispensable funding for innovative new projects. This source of funding is particularly important in our field where we have received generous corporate sponsorship for our meetings but little research support.

The remaining obstacle to progress in our specialty is commitment. While we share a deep commitment to our individual patients, our collective devotion to the research enterprise is not as apparent. It could improve in a number of ways. First, there is a limited level of participation in fundraising efforts to support musculoskeletal oncologic research. We need to develop a culture of philanthropy on the part of the physicians who treat these problems, and perhaps even more broadly across orthopaedic surgery. A sense that "we gave at the office" is not 
Symposium: 2015 Meetings of the Musculoskeletal Tumor Society and the International Society of Limb Salvage

enough. Secondly, while we pay lip service to the need for multi-institutional trials and randomized controlled trials in order to improve the quality of evidence used to direct clinical care, surgeon participation is limited. Few surgeons actually perform such trials, are willing to do the work to make the trials available in their institutions, or enroll their patients in existing trials. MSTS-sponsored and other collabora- tive trials are listed on the Society website, www.msts.org. Until the commitment to prospective research increases, our specialty will be limited to the small retrospective case series that characterize the presentations at our congresses.

While we must fight for the opportunity to be involved in fundamental basic and clinical research, we have the obligation to stay abreast of the science, clinical trials, and strategies to treat the diseases in our domain. The MSTS and ISOLS sponsor the leading forums to present new work in the field, and in collaboration with Clinical Orthopaedics and Related Research ${ }^{\circledR}$, remain in the forefront of disseminating that knowledge. Working together, we can make progress to improve the care of musculoskeletal oncology patients, as capably documented in this symposium. 